\title{
Perioperative considerations for "new" kids on the opioid block
}

\author{
Jabulani Moyo, MBChB $\cdot$ Saifee Rashiq, MB
}

Received: 2 February 2011/Accepted: 14 February 2011/Published online: 25 February 2011

(C) Canadian Anesthesiologists' Society 2011

Practicing anesthesiologists may have limited familiarity with some of the newer opioid compounds being used more frequently by medical practitioners who treat chronic pain and substance use. These drugs are interesting in their own right because of their pharmacological properties. More importantly, it is essential to understand how to care for patients who are taking these drugs to prevent harm in the perioperative context. So, what are these drugs, and what should we know about them?

\section{Tramadol}

Anyone who has either trained or practiced in Europe will be tempted to smile benignly and look down at her/his shoes when hearing tramadol being described as a "new" drug; it came into clinical use there and elsewhere in the late 1970s. Since its introduction to North America in 1995, tramadol has been marketed aggressively as being superior to traditional opioids for some types of chronic pain problems. The 2010 National Opioid Use Guidelines sanction tramadol as an appropriate choice as a first-line opioid alongside codeine. ${ }^{1}$ There is robust evidence of tramadol's effectiveness as an analgesic in several chronic pain models, including traditionally troublesome areas, such as painful neuropathy and fibromyalgia. ${ }^{2}$ Furthermore, it doesn't require the use of a triplicate prescription in many jurisdictions. When this information is added to the fact that calls for the elimination of codeine (due to

J. Moyo, MBChB · S. Rashiq, MB ( $₫)$

Division of Pain Medicine, Department of Anaesthesiology and Pain Medicine, University of Alberta, 8-120 Clinical Sciences Building, Edmonton, AB T6G 2G3, Canada

e-mail: srashiq@ualberta.ca safety concerns) are growing louder, ${ }^{3}$ it seems likely that more tramadol-treated patients will appear each year in our operating rooms and on our acute pain services.

Tramadol is a good analgesic, and its side-effect profile, particularly that relating to the gastrointestinal tract, is superior to that of codeine. Tramadol binds mu-opioid receptors, but less avidly than traditional potent opioids. Indeed, tramadol's analgesic efficacy is hard to explain if one considers its opioid actions alone; the fact that it also increases central levels of serotonin and invokes noradrenaline is an explanation for this apparent paradox. ${ }^{4}$ For the anesthesiologist, an important consideration is to estimate the relative potency of the amount of tramadol administered preoperatively in order to modify the intraoperative or postoperative doses of traditional opioids. The vigorous debates-which generally ensue when pain specialists try to agree on relative potencies between opioidsnotwithstanding, tramadol can be thought of as having about the same potency as codeine but with twice to three times the bioavailability. Tramadol also has a ceiling effect; therefore, doses exceeding $400 \mathrm{mg} \cdot$ day $^{-1}$ should not be administered. Thus, a patient who is receiving that dose can be thought of as receiving an opioid burden roughly equivalent to $1,000 \mathrm{mg} \cdot \mathrm{day}^{-1}$ of oral codeine or $30 \mathrm{mg} \cdot \mathrm{day}^{-1}$ of parenteral morphine. In Canada, tramadol is available only in oral preparations; consequently, it is not possible to use it as a sole analgesic when the patient is fasting. Preoperatively, long-acting tramadol can be continued throughout the perioperative period and can be combined with immediate-release oral tablets for the management of surgical pain. Alternatively, other immediate-release opioids can be added to the background of long-acting tramadol, or it can be discontinued safely provided that adequate amounts of traditional opioid are used to prevent pain and abstinence. ${ }^{5}$ 


\section{Tapentadol}

This drug, which has many similarities in common with tramadol, will soon be available for clinical use in Canada. Tapentadol also acts on mu receptors and inhibits reuptake of noradrenaline, but its effects on the serotonin axis are much less pronounced than that of tramadol. What are the clinical benefits of tapentadol? Unlike tramadol, proponents of the drug cite the facts that tapentadol is racemically pure and it has no active metabolites. Tapentadol acts directly on target receptors, while tramadol must be metabolized first by CYP2D6 to render it active. ${ }^{6}$ In theory, tapentadol's relative lack of effect on serotonin physiology ought to lower the risk of serotonin syndrome (the reason why tramadol and selective serotonin reuptake inhibitors or monoamine oxidase inhibitors may not be taken concurrently). There is certainly evidence that tapentadol is an effective analgesic and that it has, as one would expect, a better side-effect profile than older agents. $^{7,8}$ To our knowledge, there are no trials in which tramadol and tapentadol are compared directly for efficacy and side effects, and tapentadol is unlikely to offer a price advantage over tramadol, which can now be manufactured generically. Like pregabalin, we anticipate that the degree to which tapentadol is embraced by the pain management community will owe as much or more to the way in which the drug is marketed as to its intrinsic advantages over available therapies. In the meantime, anesthesiologists who encounter early adopters of the drug in the perioperative setting or on the acute pain service might find it helpful to remember that tapentadol $50 \mathrm{mg}$ po is roughly equipotent to oxycodone $10 \mathrm{mg} p o$, and it can be discontinued with impunity provided enough alternative opioid is supplied to prevent abstinence.

\section{Buprenorphine}

Buprenorphine is another vintage drug that took some time to find its way to our shores, and it is the new arrival with the greatest potential to generate confusion in the perioperative setting. Buprenorphine is available in two forms, as transdermal patches for use in chronic pain management and as sublingual tablets for maintenance treatment in opioid addiction.

The strength of the transdermal patches available in Canada is lower ( 5 to $20 \mu \mathrm{g} \cdot \mathrm{hr}^{-1}$ ) than the strength of those that have been used in Europe (up to $70 \mu \mathrm{g} \cdot \mathrm{hr}^{-1}$ ) for many years. The attractions of this drug over traditional opioids include a lower incidence of gastrointestinal side effects, a decreased risk of respiratory depression, no interference with gonadotrophin axes, no immunoparesis, and an intrinsic antihyperalgesic property. ${ }^{9}$ Having observed that the drug exhibits a ceiling for respiratory depression, early studies concluded that it had partial agonistic activity at the mu receptor. This conclusion was later discounted because it was based on the apparently reasonable assumption that this ceiling effect would be observed in all effects modulated by the same receptor. In fact, there is no ceiling for buprenorphine analgesia, and it has been used to good effect even in severe cancer and non-cancer pain lasting many months. ${ }^{10-12}$ This apparent paradox, i.e., unlimited analgesic potential combined with a ceiling on adverse effects, sounds almost too good to be true. The explanation may lie in the observation that buprenorphine has a differential effect, which results in its being more potent at spinal receptor sites than at cerebral opioid receptor sites, the latter obviously being where centrally-mediated adverse effects would be generated.

Buprenorphine sublingual tablets, both in the pure drug and in a 4:1 combination with naloxone, are intended for the maintenance treatment of opioid addiction. ${ }^{13}$ The drug is equally effective for that purpose either with or without naloxone, but the latter has been added to the latest product, Suboxone ${ }^{\circledR}$, to discourage its intravenous injection. Those who treat addiction have found many reasons to like this compound; it binds very avidly to mu receptors and therefore occupies them for a long time, thus effectively preventing abstinence phenomena. It is not detected by standard urine tests for opioids, making the appearance of opioids in drug screens less difficult to interpret. The very slow dissociation of buprenorphine from mu receptors means that the drug can be dosed as infrequently as every other day, which, for the first time, enables safe maintenance treatment to be given without the necessity for daily attendance. Withdrawal symptoms following discontinuation of buprenorphine are said to be much less severe and prolonged than those experienced following morphine and its analogues or methadone.

The potential trap for anesthesiologists looking after patients who are taking buprenorphine is getting enough potent opioid to bind to the receptor to provide sufficient analgesia for surgical pain. The high affinity of the drug for the mu receptor, combined with the very slow dissociation of the drug-receptor complex, makes this a potentially difficult undertaking. ${ }^{13}$ For elective surgery, preemptive rotation from buprenorphine to a traditional potent mu agonist, such as hydromorphone, makes sense if there is time. When a patient on buprenorphine must have emergency surgery, an ad hoc analgesia plan in which effective non-opioid techniques, such as regional blockade, non-steroidal antiinflammatory drugs, and gabapentin, are combined with a traditional opioid (potentially in higher doses and with careful monitoring) may be the best option. In any event, the situation should improve from day to day as buprenorphine slowly loosens its grip on its target receptor. 
Theoretically, although buprenorphine ought to be an attractive choice as a stand-alone perioperative analgesic, this is best avoided by those who are not experienced in prescribing the drug. The administration of buprenorphine to a person relying on some degree of receptor occupancy by a traditional opioid for comfort could result in the precipitation of withdrawal by displacement of the first drug. ${ }^{14}$ Although its receptor occupancy is prolonged, those who prescribe sublingual buprenorphine as an analgesic must be mindful of the fact that its analgesic duration of action is only six to eight hours. This will sound strangely familiar to those who prescribe methadone for chronic pain, but it should not imply that familiarity with methadone ipso facto confers the skills necessary to use the newer drug safely.

Rational adoption of new drugs into clinical practice should be based on improvements in efficacy, favourable safety and side effect profiles, and overall cost-effectiveness. Tramadol is a better drug than codeine for adults with mild to moderate acute and chronic pain, and in most circumstances, tramadol should replace codeine for that purpose provided the patient can afford to have the prescription filled. Transdermal buprenorphine is a serious contender as a first-line opioid in ambulatory patients with chronic pain, particularly in patients who are predicted to have a high risk of side effects from more traditional opioids. However, the relative lack of potency of the patches marketed in Canada will render it less useful here than it has been in Europe. Sublingual buprenorphine provides addiction medicine practitioners with a number of important new treatment options, and its use will become more widespread for that purpose. Increasingly, anesthesiologists will need a working knowledge of these drugs, and excellent reviews are available for this purpose., ${ }^{4,6,15}$ Meanwhile, we all must continue to wait for the one tool we really need to make the prescribing of opioids completely safe, i.e., a potent, versatile and well-tolerated analgesic that isn't an opioid at all.

\section{Considérations périopératoires concernant les 'nouveaux' venus chez les opioïdes}

Les anesthésiologistes en pratique ont peut-être une connaissance limitée des opiö̈des utilisés le plus fréquemment par les médecins qui traitent les douleurs chroniques et l'abus de substances. Cependant, en raison de leurs propriétés pharmacologiques, ces médicaments méritent notre intérêt. Et, ce qui est plus important encore, c'est qu'il est essentiel de comprendre quels soins prodiguer aux patients qui prennent ces médicaments si l'on souhaite prévenir les incidents dans un cadre périopératoire. Dès lors, quels sont ces médicaments, et que devrait-on savoir à leur propos?

\section{Le tramadol}

Toute personne ayant été formée ou ayant pratiqué en Europe sera tentée de sourire avec bienveillance et de regarder ailleurs lorsqu'elle entend le tramadol décrit comme un «nouveau » médicament; en effet, son utilisation clinique là-bas et ailleurs remonte à la fin des années 1970. Depuis son introduction en Amérique du Nord en 1995, le tramadol a fait l'objet d'une commercialisation agressive comme étant supérieur aux opioïdes traditionnels pour traiter certains types de problèmes liés à la douleur chronique. Les lignes directrices nationales sur l'utilisation des analgésiques opioïles de 2010 considèrent le tramadol, tout comme la codéine, comme étant un choix adapté comme opioïde de première intention. ${ }^{1}$ Des données probantes rigoureuses soutiennent l'efficacité du tramadol comme analgésique dans plusieurs modèles de douleur chronique, y compris dans des domaines traditionnellement problématiques comme les neuropathies douloureuses et la fibromyalgie. ${ }^{2}$ En outre, dans plusieurs juridictions, le tramadol ne requiert pas une ordonnance en trois exemplaires. Lorsqu'on ajoute à cela l'appel croissant à l'élimination de la codéine (pour des raisons de sécurité), ${ }^{3}$ il semble donc probable que le nombre de patients traités avec le tramadol ira croissant au fil des ans dans nos salles d'opération et nos services d'analgésie postopératoire.

Le tramadol est un bon analgésique, et son profil d'effets secondaires, particulièrement ceux liés au tractus gastro-intestinal, est supérieur à celui de la codéine. Le tramadol se lie aux récepteurs opioïdes mu, mais avec moins d'avidité que les opioïdes puissants conventionnels. En effet, l'efficacité analgésique du tramadol est difficile à expliquer si l'on ne tient compte que de son action opioïde; néanmoins, ce paradoxe apparent peut s'expliquer par le fait que cet agent augmente également les niveaux centraux de sérotonine et sollicite la noradrénaline. ${ }^{4}$ Pour l'anesthésiologiste, il est important d'estimer la puissance relative de la quantité de tramadol administrée avant l'opération afin de modifier les doses per- et postopératoires d'opioïdes traditionnels. Nonobstant les débats animés - qui font en général rage lorsque des spécialistes de la douleur tentent de se mettre d'accord sur les puissances relatives des différents opiö̈des -, le tramadol peut être considéré comme possédant plus ou moins la même puissance que la codéine, mais deux à trois fois sa biodisponibilité. Le tramadol a également un effet plafond, c'est pourquoi des doses supérieures à 
$400 \mathrm{mg} \cdot$ jour $^{-1}$ ne devraient pas être administrées. Ainsi, on peut considérer un patient qui reçoit cette dose comme recevant un fardeau opioïde approximativement équivalent à $1000 \mathrm{mg} \cdot$ jour $^{-1}$ de codéine orale, ou $30 \mathrm{mg} \cdot$ jour $^{-1}$ de morphine parentérale. Au Canada, le tramadol n'est disponible qu'en préparation orale; par conséquent, il ne peut être utilisé comme analgésique unique lorsque le patient jeûne. En période peropératoire, le tramadol à action prolongée peut être maintenu tout au long de la période périopératoire et peut être combiné à des comprimés oraux à libération immédiate pour contrôler la douleur chirurgicale. Une autre possibilité est d'ajouter d'autres opioïdes à libération immédiate à la base de tramadol à action prolongée; le tramadol peut également être interrompu en toute sécurité, tant que des quantités adaptées d'opioïdes traditionnels sont utilisées pour prévenir la douleur et l'abstinence. ${ }^{5}$

\section{Le tapentadol}

Ce médicament, qui partage plusieurs similarités avec le tramadol, sera bientôt disponible pour une utilisation clinique au Canada. Le tapentadol agit également sur les récepteurs mu et inhibe le recaptage de la noradrénaline, mais ses effets sur l'axe de sérotonine sont beaucoup moins prononcés que ceux du tramadol. Quels sont les avantages cliniques du tapentadol? Les défenseurs de ce médicament mentionnent que contrairement au tramadol, le tapentadol possède une pureté racémique et aucun métabolite actif. Le tapentadol agit directement sur les récepteurs cibles, alors que le tramadol doit d'abord être métabolisé par le CYP2D6 pour devenir actif. ${ }^{6}$ En théorie, l'absence relative d'effet du tapentadol sur la physiologie de la sérotonine devrait réduire le risque de syndrome sérotoninergique - la raison pour laquelle le tramadol et certains inhibiteurs du recaptage de la sérotonine ou inhibiteurs de la monoamine-oxydase ne peuvent pas être pris simultanément. Selon certaines données probantes, le tapentadol est un analgésique efficace et possède, sans surprise, un meilleur profil d'effets secondaires que les agents plus anciens. ${ }^{7,8} \grave{A}$ notre connaissance, il n'existe pas d'études comparant directement l'efficacité et les effets secondaires du tramadol et du tapentadol, et il est peu probable que le tapentadol soit moins dispendieux que le tramadol, lequel est actuellement fabriqué sous forme générique. Tout comme cela a été le cas avec la prégabaline, nous prévoyons que le degré d'adoption du tapentadol par les personnes traitant la douleur dépendra tout autant, voire plus, de la façon dont ce médicament sera commercialisé que de ses avantages intrinsèques par rapport à d'autres traitements. En attendant, il peut être utile aux anesthésiologistes côtoyant des personnes ayant déjà adopté le médicament dans un contexte périopératoire ou dans le service d'analgésie postopératoire de se souvenir que $50 \mathrm{mg}$ po de tapentadol sont plus ou moins équipotents à $10 \mathrm{mg}$ po d'oxycodone, et qu'il peut être interrompu en toute impunité si un autre opioïde en quantité suffisante est administré pour empêcher l'abstinence.

\section{La buprénorphine}

La buprénorphine est un autre 'vieux' médicament qui a pris son temps avant d'accoster sur nos rivages. Il s'agit du nouvel arrivé affichant le plus grand potentiel de créer la confusion dans un cadre périopératoire. La buprénorphine est disponible sous deux formes, soit en timbre transdermique qui peut être utilisé pour la prise en charge de la douleur chronique, soit en comprimé sublingual pour le traitement d'entretien d'une dépendance aux opioïdes.

La puissance des timbres transdermiques disponibles au Canada est moins élevée (5 à $20 \mu \mathrm{g} \cdot \mathrm{h}^{-1}$ ) que celle des timbres utilisés depuis de nombreuses années en Europe (jusqu'à $70 \mu \mathrm{g} \cdot \mathrm{h}^{-1}$ ). Parmi les attraits de ce médicament par rapport aux opiö̈des conventionnels, citons une incidence plus faible d'effets secondaires gastro-intestinaux, un risque réduit de dépression respiratoire, aucune modification des axes de la gonadotrophine, aucune immunoparésie, et une propriété antihyperalgésique intrinsèque. ${ }^{9}$ En observant que ce médicament affiche un plafond pour la dépression respiratoire, les premières études ont conclu qu'il possédait une activité agoniste partielle au récepteur mu. Cette conclusion a par la suite été réfutée parce qu'elle se fondait sur la présomption, raisonnable en apparence, que cet effet plafond serait observé dans tous les effets modulés par le même récepteur. En fait, il n'y a pas de plafond pour l'analgésie avec la buprénorphine, et ce médicament a été utilisé de façon concluante dans des cas de douleur cancéreuse et non cancéreuse grave durant plusieurs mois. ${ }^{10-12}$ Ce paradoxe apparent, soit un potentiel analgésique illimité combiné à un plafond des effets indésirables, a l'air trop beau pour être vrai. L'explication pourrait se trouver dans le fait que la buprénorphine a un effet différentiel, d'où sa puissance supérieure aux sites de récepteurs rachidiens qu'aux sites de récepteurs opioïdes cérébraux, ces derniers étant évidemment le site de génération des effets indésirables médiés par le système nerveux central.

L'utilisation prévue des comprimés sublinguaux de buprénorphine, sous forme d'agent pur ou de combinaison à 4:1 avec de la naloxone, est le traitement d'entretien des dépendances aux opioïdes. ${ }^{13}$ Avec ou sans naloxone, ce médicament est tout aussi efficace pour cette indication. Toutefois, la naloxone a été ajoutée au produit le plus récent, le Suboxone ${ }^{\circledR}$, afin de décourager son injection 
intraveineuse. Les personnes traitant les dépendances ont trouvé nombre de raisons d'apprécier cette molécule; elle se lie avec avidité aux récepteurs mu et les occupe par conséquent pour une période prolongée, prévenant par là même, de manière efficace, les phénomènes d'abstinence. La buprénorphine n'est pas détectée par les tests standards d'urine cherchant la présence d'opioïdes, ce qui rend la présence d'opioïdes dans les tests de dépistage de drogue plus facile à interpréter. En raison de la dissociation très lente de la buprénorphine des récepteurs mu, cet agent peut être administré aussi peu souvent qu'un jour sur deux - en d'autres termes, il s'agit du premier traitement d'entretien sécuritaire qui peut être donné sans requérir une présence quotidienne. Les symptômes de sevrage provoqués par l'interruption d'un traitement à la buprénorphine sont, semble-t-il, beaucoup moins prononcés et d'une durée moindre que ceux suivant l'interruption de la morphine et de ses analogues, ou de la méthadone.

Le piège potentiel pour les anesthésiologistes s'occupant de patients prenant de la buprénorphine est d'obtenir suffisamment d'opioïdes puissants qui se lient aux récepteurs afin de procurer une analgésie suffisante pour la douleur chirurgicale. L'affinité élevée de cet agent pour le récepteur mu, combinée à la dissociation très lente du complexe médicament-récepteur, rend ce défi potentiellement ardu. ${ }^{13}$ Pour les chirurgies non urgentes, le remplacement préventif de la buprénorphine par un agoniste mu puissant conventionnel, tel que l'hydromorphone, est logique lorsqu'il y a suffisamment de temps. Lorsqu'un patient sous buprénorphine doit subir une chirurgie d'urgence, un plan d'analgésie $a d$ hoc, combinant des techniques non opioïdes efficaces telles qu'un bloc régional, des anti-inflammatoires non stéroïdiens et de la gabapentine à un opioïde conventionnel (potentiellement en doses plus fortes et avec un monitorage attentif), pourrait être la meilleure option. Dans tous les cas, la situation devrait s'améliorer de jour en jour, au fur et à mesure que la buprénorphine se détache lentement de son récepteur cible.

En théorie, alors que la buprénorphine devrait être un choix séduisant comme analgésique périopératoire unique, il vaut mieux que les praticiens sans expérience dans la prescription de ce médicament l'évitent. L'administration de buprénorphine à une personne qui dépend d'une certaine occupation des récepteurs par un opioïde traditionnel pour son confort pourrait précipiter le sevrage en supplantant le premier médicament. ${ }^{14}$ Bien que l'occupation des récepteurs soit prolongée avec la buprénorphine, les personnes la prescrivant comme analgésique sous sa forme sublinguale doivent garder à l'esprit que sa durée d'action analgésique n'est que de six à huit heures. Cela rappellera certainement des souvenirs à ceux qui prescrivent de la méthadone pour le traitement de la douleur chronique, mais cela ne veut pas dire qu'une connaissance des propriétés de la méthadone confère par le fait même les compétences nécessaires à l'utilisation sécuritaire de ce nouvel agent.

L'adoption rationnelle de nouveaux agents dans la pratique clinique devrait se fonder sur les améliorations qu'ils apportent au niveau de leur efficacité, de leurs profils d'innocuité et d'effets secondaires favorables, et de leur rentabilité globale. Le tramadol est un meilleur agent que la codéine pour les adultes souffrant de douleur chronique ou aiguë légère à modérée et, dans la plupart des situations, il devrait la remplacer pour cette indication - pour autant que le patient ait les moyens de payer le médicament prescrit. La buprénorphine transdermique est un compétiteur sérieux en tant qu'opioïde de première intention chez les patients en ambulatoire souffrant de douleur chronique, et particulièrement chez les patients pour lesquels on prédit un risque élevé d'effets secondaires s'ils prenaient des opioïdes plus conventionnels. Néanmoins, le manque relatif de puissance des timbres commercialisés au Canada réduira leur utilité ici par rapport à l'Europe. La buprénorphine sublinguale offre aux médecins traitant les dépendances plusieurs nouvelles options thérapeutiques, et son utilisation ira grandissant pour cette indication. Les anesthésiologistes auront de plus en plus besoin d'une connaissance pratique de ces agents, et d'excellents comptes rendus sont à leur portée pour se familiariser avec ces agents. ${ }^{4,6,15}$ Entre-temps, nous devons tous continuer à attendre l'outil dont nous avons réellement besoin pour rendre la prescription d'opioïdes entièrement sécuritaire: un analgésique puissant, polyvalent et bien toléré - qui n'est pas un opioïde.

Conflicts of interest Dr. Saifee Rashiq recruited participants for two manufacturer-sponsored clinical trials of transdermal buprenorphine. That association ended in 2007.

\section{References}

1. National Opioid Use Guideline Group (NOUGG). Canadian Guideline for Safe and Effective Use of Opioids for Chronic NonCancer Pain. 2010. Available from URL: Nationalpaincentre. mcmaster.ca/opioid/ (accessed February 2011).

2. Rosenberg T. The role of Tramadol ER in the treatment of chronic pain. Int J Clin Pract 2009; 63: 1531-43.

3. MacDonald N, Macleod SM. Has the time come to phase out codeine? CMAJ 2010; 182: 1825.

4. Leppert $W$. Tramadol as an analgesic for mild to moderate cancer pain. Pharmacol Rep 2009; 61: 978-92.

5. Di Lorenzo L, Foti C, Forte AM, et al. The addition of tramadol as a second opioid may improve pain relief in severe osteoarthritis: a prospective study. Pain Pract 2010; 10: 540-7.

6. Tzschentke TM, Christoph T, Kogel B, et al. (-)-(1R, 2R)-3-(3dimethylamino-1-ethyl-2-methyl-propyl)-phenol hydrochloride (tapentadol $\mathrm{HCl}$ ): a novel u-opioid receptor agonist/norepinephrine reuptake inhibitor with broad-spectrum analgesic properties. J Pharmacol Exp Ther 2007; 323: 265-76. 
7. Hartrick C, Van Hove I, Stegmann JU, Oh C, Upmalis D. Efficacy and tolerability of tapentadol immediate release and oxycodone $\mathrm{HCl}$ immediate release in patients awaiting primary joint replacement surgery for end-stage joint disease: a 10-day, phase III, randomized, double-blind, active and placebo-controlled study. Clin Ther 2009; 31: 260-71.

8. Wild JE, Grond S, Kuperwasser B, et al. Long term safety and tolerability of tapentadol extended release for the management of chronic low back pain or osteoarthritis pain. Pain Pract 2010; 10: 416-27.

9. Pergolizzi J, Aloisi AM, Dahan A, et al. Current knowledge of buprenorphine and its unique pharmacological profile. Pain Pract 2010; 10: 428-50.

10. Sittl R, Likar R, Nautrup PB. Equipotent doses of transdermal fentanyl and transdermal buprenorphine in patients with cancer and noncancer pain: results of a prospective cohort study. Clin Ther 2005; 27: 225-37.
11. Likar $R$, Sittl $R$. Transdermal buprenorphine for treating nociceptive and neuropathic pain: four case studies. Anesth Analg 2005; 100: 781-5.

12. Dahan A, Yassen A, Romberg R, et al. Buprenorphine induces ceiling effect in respiratory depression but not in analgesia. Br J Anaesth 2006; 96: 627-32.

13. Vadivelu N, Anwar M. Buprenorphine in postoperative pain management. Anesthesiol Clin 2010; 28: 601-9.

14. Oifa S, Sydoruk T, White I, et al. Effects of intravenous patientcontrolled analgesia with buprenorphine and morphine alone and in combination during the first 12 postoperative hours: a randomized, double blind, four-arm trial in adults undergoing abdominal surgery. Clin Ther 2009; 31: 527-41.

15. Kress $H G$. Clinical update on pharmacology, efficacy and safety of transdermal buprenorphine. Eur J Pain 2009; 13: 219-30. 